\title{
Evolución clínica de pacientes chilenos con tirosinemia tipo I tratados con 2-(2-nitro-4-trifluorometilbenzoil)- 1,3-ciclohexanediona (NTBC)
}

\author{
ERNA RAIMANN, VERÓNICA CORNEJO ${ }^{\mathrm{a}, \mathrm{b}}$, CAROLINA ARIAS, \\ JUAN FRANCISCO CABELLO, GABRIELA CASTRO ${ }^{a}$, \\ ELOINA FERNÁNDEZ ${ }^{\mathrm{a}}$, ALICIA DE LA PARRA ${ }^{\mathrm{c}}$
}

\section{Clinical follow up of Chilean patients with tyrosinemia type 1 treated with 2-(2-nitro-4-trifluoromethylbenzoyl)- 1,3-ciclohexanedione (NTBC)}

Background: Tyrosinemia type I is an inborn error of metabolism due to deficiency of fumarilacetoacetase. Acute presentation is with liver failure, hypophosphatemic rickets and peripheral neuropathy. Chronic presentation is with visceromegaly and subclinical rickets. The most severe complications are hepatic cancer and acute neurological crises. Without treatment, tyrosinemia type 1 is fatal. In 1992 treatment for tyrosinemia type 1 with 2-(2-nitro-4-trifluoromethybenzoyl)-1,3-ciclohexanedione (NTBC) was proposed. A clinical response was reported in $90 \%$ of patients. In cases that did not respond, a successful liver transplantation was performed, reducing mortality to 5\%. Aim: To report the follow up of 12 patients treated with NTBC. Patients and Methods: Review of clinical records of 12 Chilean cases treated with NTBC at the Instituto de Nutrición y Tecnología de los Alimentos (INTA) from January 2004 until June 2010. Results: In all patients, a rapid metabolic control was achieved. Two patients developed hepatocarcinoma. One of these patients died and one was successfully treated with liver transplantation. One patient died after receiving a liver transplantation. Nine patients have at present good liver function, but 2 had peripheral neuropathy due to late diagnosis and discontinuing NTBC treatment. Conclusions: Treatment with NTBC allows metabolic normalization in tyrosinemia type 1, prevents liver cirrhosis and hepatic cancer, improving survival rates and quality of life in the patients. Neonatal screening is essential for the early diagnosis of this treatable disease, that otherwise may be lethal.

(Rev Med Chile 2012; 140: 169-175).

Key words: Cyclohexanones; Liver neoplasms; Tyrosinemias.
Laboratorio de genética y enfermedades metabólicas, Instituto de Nutrición y Tecnología de los Alimentos (INTA), Universidad de Chile.

aLicenciada en Nutrición. ${ }^{b} M S c$ Nutrición. 'Psicóloga.

Apoyo financiero: NTBC donado por Laboratorio Orphan ${ }^{\circledR}$

Recibido el 10 de agosto de 2011, aceptado el 27 de octubre de 2011.

Correspondencia a: Dra. Erna Raimann INTA, Universidad de Chile El Líbano 5524, Santiago, Chile. E-mail: eraimann@inta. uchile.cl
L

a tirosinemia tipo I es un error innato del metabolismo, caracterizado por el déficit lándose fumarilacetoacetato y maleilacetoacetato, causantes de daño hepático y renal. Consecuentemente se produce succinilacetona, cuya medición permite hacer el diagnóstico (Figura 1). La incidencia de la enfermedad en la población mundial es $1 / 100.000$ nacidos vivos ${ }^{1-5}$.

Existe una gran heterogeneidad clínica, pudiendo manifestarse desde lactante hasta la edad adulta. La forma aguda se caracteriza por insuficiencia 
hepática y hepatomegalia; pudiendo desarrollar raquitismo hipofosfémico con síndrome de Fanconi, polineuropatía y dolor abdominal similar al descrito en la porfiria aguda intermitente, debido a que succinilacetona produce inhibición enzimática de porfobilinógeno sintetasa. En la forma crónica la sintomatología es menos llamativa con leve visceromegalia, raquitismo subclínico y moderado retardo del crecimiento. Una de las complicaciones más graves es el hepatocarcinoma, que se ha encontrado desde el primer año de vida, reportándose en $37 \%$ de los niños mayores de 2 años portadores de esta enfermedad ${ }^{1,4,6,7}$. La tirosinemia tipo I, si no se trata, tiene pronóstico fatal, tanto en su forma aguda como crónica. Esta evolución natural fue transformada con el uso del trasplante hepático. Sin embargo, la cirrosis hepática y la displasia hepatocelular se pueden reproducir en el hígado trasplantado, en algunos casos antes de 2 años $^{6,7}$. Tradicionalmente el tratamiento ha sido una dieta con restricción de aminoácidos, fenilalanina y tirosina, que mejora la función renal, pero no evita el hepatocarcinoma y la neuropatía periférica.

En 1992, Lindstedt et al propusieron el uso de NTBC (2-(2-nitro-4-trifluorometilbenzoil)1,3-ciclohexanediona), inhibidor de la enzima 4-hidroxifenilpiruvato dioxigenasa, como terapia alternativa para tirosinemia tipo 1 (Figura 1). El estudio realizado por estos autores mostró una respuesta bioquímica inmediata, con disminución de succinilacetona en orina a las 12 horas de administrado NTBC, hasta hacerse indetectable ${ }^{8-10}$. En 1998 se informó los resultados de este mismo proyecto, que mostraban que $90 \%$ de los pacientes había respondido clínicamente al tratamiento con NTBC. En la mitad de los que no respondieron, se realizó trasplante hepático exitosamente con reducción de la tasa de mortalidad a 5\%. El estudio mostró una disminución del riesgo de desarrollo precoz de hepatocarcinoma en los que iniciaban tratamiento a edad temprana. Aquellos que habían iniciado NTBC antes de los 2 meses de edad no desarrollaron hepatocarcinoma a los 2 años de tratamiento ${ }^{11,12}$.

En Chile han sido tratados desde 1996 doce pacientes con NTBC a través del Proyecto Multicéntrico Internacional NTBC (Holme and Lindstedt ${ }^{12}$.

El objetivo de este artículo es dar a conocer la presentación y evolución de los pacientes con tirosinemia tipo I controlados en el INTA, Universidad de Chile, que han sido seguidos de acuerdo a las normas establecidas por Proyecto Multicéntrico Internacional NTBC.

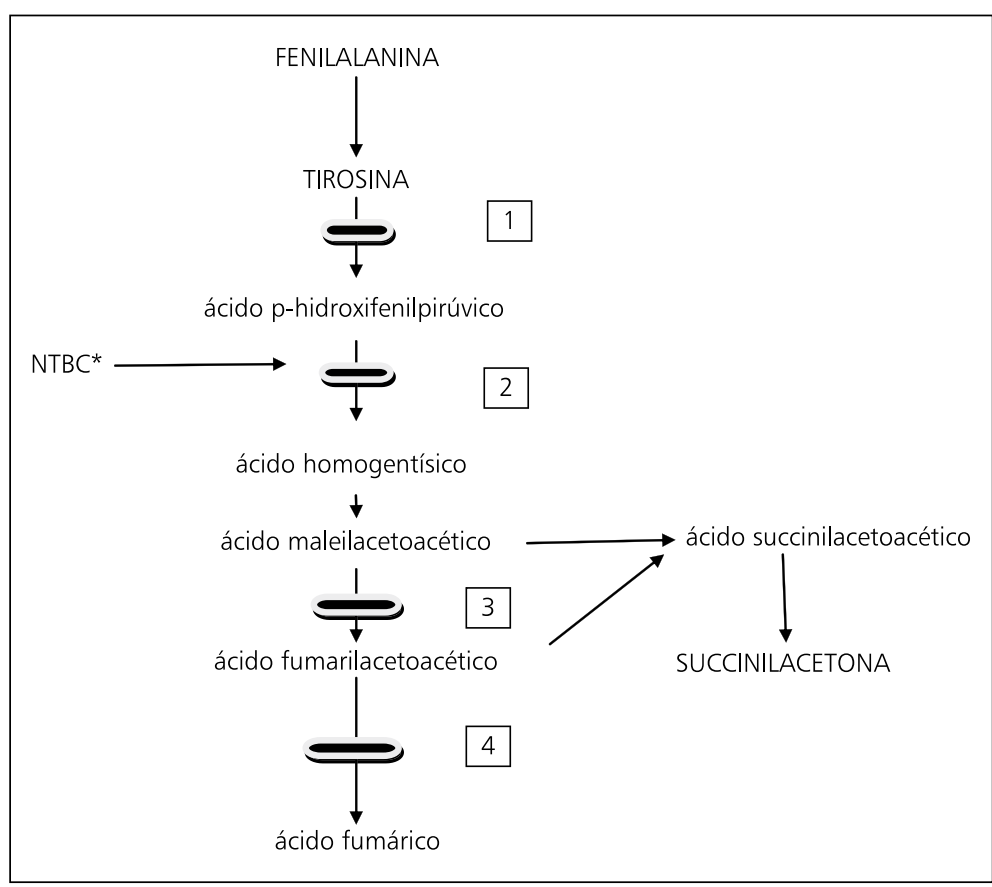

Figura 1. Vía metabólica tirosina y sitio bloqueo NTBC: 1) tirosina aminotransferasa, TIROSINEMIA II; 2) 4-OH-piruvato dioxigenasa, TIROSINEMIA III (* inhibida por el NTBC); 3) fumarilacetoacetato hidrolasa, TIROSINEMIA Ia; 4) Maleilacetoacetato isomerasa, TROSINEMIA Ib. 


\section{Pacientes y Método}

Se revisaron las fichas clínicas de los pacientes tratados en el Laboratorio de Enfermedades Metabólicas del INTA, en forma retrospectiva desde enero 2004 hasta junio 2010. El primer paciente ingresó al protocolo en 1996 y el último en el año 2009. En la Tabla 1 se detalla el tiempo de seguimiento de cada sujeto. Se incluyeron en este estudio todos los casos a los que se les confirmó el diagnóstico de tirosinemia tipo I con medición de succinilacetona en orina realizada en la Universidad de Göteborg; siendo éste el único criterio de inclusión, no hubo criterios de exclusión.

Se siguieron 12 portadores de tirosinemia tipo I, de ellos 8 mujeres y 4 hombres. Desde el momento de la confirmación diagnóstica todos recibieron NTBC en la dosis sugerida de $1 \mathrm{mg} /$ $\mathrm{kg}$ /día, la que se da por vía oral cada 8 horas y dieta con restricción de aminoácidos fenilalanina y tirosina. La adherencia al tratamiento se evaluó con concentraciones plasmáticas de NTBC y entrevista nutricional realizada por nutricionista especializada. Desde el inicio de tratamiento los pacientes fueron controlados cada 2 meses el primer año y posteriormente cada 3 meses. Los controles se realizaron por nutricionista, pediatra y neurólogo infantil del laboratorio. También se les realizó evaluación de desarrollo psicomotor con Escala de Bayley II y Coeficiente Intelectual (CI) con la Prueba WISC-R, dependiendo de la edad, por psicóloga del laboratorio. Los padres firmaron un consentimiento informado para ingresar al Protocolo NTBC diseñado por Proyecto NTBC, previamente validado por Comité de Ética de la Universidad de Göteborg y por el Comité de Ética

Tabla 1. Características de los pacientes con tirosinemia al inicio de tratamiento y estado actual

\begin{tabular}{|c|c|c|c|c|c|}
\hline Enfermo & $\begin{array}{l}\text { Edad primeros } \\
\text { síntomas }\end{array}$ & $\begin{array}{l}\text { Edad inicio } \\
\text { Tratamiento }\end{array}$ & $\begin{array}{l}\text { Tiempo de } \\
\text { seguimiento }\end{array}$ & $\begin{array}{l}\text { Presentación } \\
\text { clínica }\end{array}$ & Estado actual \\
\hline 1 & 1 mes & 3 meses & $\begin{array}{l}14 \text { años } \\
2 \text { meses }\end{array}$ & Falla hepática aguda & $\begin{array}{l}\text { Bien. } \\
\text { Cl limítrofe }\end{array}$ \\
\hline 2 & 1 año & 5 años & 4 años & Pseudoporfiria & $\begin{array}{l}\text { Falleció por } \\
\text { Hepatocarcinoma }\end{array}$ \\
\hline 3 & 1 año & 4 años & $\begin{array}{l}11 \text { años } \\
9 \text { meses }\end{array}$ & Raquitismo & Bien, limítrofe \\
\hline 4 & 16 meses & 17 meses & $\begin{array}{l}2 \text { años } \\
2 \text { meses }\end{array}$ & $\begin{array}{l}\text { Insuf. hepática aguda } \\
\text { Raquitismo }\end{array}$ & Falleció post-trasplante \\
\hline 5 & 1 mes & 9 meses & $\begin{array}{l}8 \text { años } \\
6 \text { meses }\end{array}$ & Raquitismo & Bien. Limítrofe \\
\hline 6 & 8 meses & 10 años & $\begin{array}{l}6 \text { años } \\
6 \text { meses }\end{array}$ & $\begin{array}{l}\text { Raquitismo, } \\
\text { Neuropatía periférica }\end{array}$ & $\begin{array}{l}\text { Talla baja, } \\
\text { Hepatocarcinoma, } \\
\text { Trasplante hepático }\end{array}$ \\
\hline 7 & 1 mes & 2 meses & $\begin{array}{l}8 \text { años } \\
8 \text { meses }\end{array}$ & Falla hepática & $\begin{array}{l}\text { Bien, retardo mental } \\
\text { leve }\end{array}$ \\
\hline 8 & 8 meses & 9 meses & $\begin{array}{l}6 \text { años } \\
8 \text { meses }\end{array}$ & $\begin{array}{l}\text { Falla hepática aguda } \\
\text { Raquitismo }\end{array}$ & Bien, Cl normal \\
\hline 9 & 4 meses & 11 meses & $\begin{array}{l}6 \text { años } \\
8 \text { meses }\end{array}$ & Falla hepática & Bien, Cl normal \\
\hline 10 & 3 meses & 3 meses & $\begin{array}{l}5 \text { años } \\
2 \text { meses }\end{array}$ & Falla hepática & $\begin{array}{l}\text { Bien. } \\
\text { Limítrofe }\end{array}$ \\
\hline 11 & 2 meses & 2 meses & $\begin{array}{l}5 \text { años } \\
3 \text { meses }\end{array}$ & Falla hepática & Bien, Cl normal \\
\hline 12 & 6 meses & 7 meses & 18 meses & Falla hepática & $\begin{array}{l}\text { Bien, desarrollo } \\
\text { psicomotor normal }\end{array}$ \\
\hline
\end{tabular}


del INTA, Universidad de Chile. No hubo ninguno que rechazara el ingreso a este proyecto.

Se realizó un estudio de corte descriptivo retrospectivo, analizando las variables, al momento del diagnóstico, al inicio de tratamiento, al mes y luego cada 6 meses hasta el tiempo actual. Debemos destacar la adherencia que presentaron estos pacientes al seguimiento.

Se evaluó estado nutricional con $\mathrm{Z}$ score $\mathrm{P} / \mathrm{T}$, albuminemia, proteínas totales; desarrollo psicomotor o coeficiente intelectual. Para medir función hepática se analizó protrombinemia, bilirrubina total, transaminasas, fosfatasas alcalinas, $\alpha$-feto proteína. La función renal fue valorada mediante creatininemia, nitrógeno ureico, calcemia y fosfemia. El control metabólico de la enfermedad se determinó con eritroporfobilinógeno sintetasa, succinilacetona (plasma y orina), ácido 5 aminolevulínico.

No se registraron reacciones adversas atribuibles a NTBC

\section{Análisis estadístico}

Dado el bajo número de pacientes, por lo infrecuente de esta patología, se realizó análisis de frecuencia y promedio de las variables estudiadas con curva evolutiva individual.

\section{Resultados}

Todos los pacientes estudiados eran chilenos, salvo 1 que es peruano, 8 mujeres y 4 hombres.

La forma clínica de presentación fue variada, 7/12 con hepatoesplenomegalia, 5/12 con insuficiencia hepática, $5 / 12$ con raquitismo hipofosfémico (síndrome de Fanconi), 3/12 con falla hepática progresiva, $1 / 12$ con crisis de dolor abdominal semejante a porfiria aguda. Las edades de presentación de los primeros síntomas fueron de 0 a 6 meses: 7 casos, de 6 a 12 meses: 4 y de 12 a 48 meses: 1 (Tabla 1). Estos datos fueron obtenidos por anamnesis, identificándose síntomas precoces atribuibles a la enfermedad.

Las edades de diagnóstico presentaron la siguiente distribución: de 0-6 meses 4 pacientes, de 6-12 meses 4, de 12-48 meses 1, de 2-6 años 2 y un caso a los 10 años. Llama la atención que pese a que los síntomas se presentaron en todos los afectados antes de los 2 años de vida, en algunos el diagnóstico tardó varios años, siendo llamativa la paciente que inició síntomas a los 8 meses y se diagnosticó a los 10 años. El primer caso ha sido seguido por 14 años y el último tiene 18 meses de tratamiento.

A todos los pacientes se les realizó espectrometría de masas en tandem en el momento de ser derivados al INTA, la que mostró aumento de tirosina, fenilalanina y metionina, promedio de tirosina $299 \mathrm{mM} / \mathrm{l}$ (Valor Normal 0-121). Con este patrón de acilcarnitinas más la clínica se planteó una probable tirosinemia tipo 1 y se confirmó el diagnóstico con succinilacetona en orina con un promedio de $523 \mathrm{mM} /$ Mcreat $(\mathrm{VN}<1)$.

\section{Evolución clínica antes del diagnóstico}

Todos los pacientes presentaron función hepática anormal al inicio, previo al tratamiento, caracterizada por alteración de pruebas hepáticas, sin colestasis, predominando la hipoprotrombinemia. Todos evolucionaron con desnutrición en distinto grado y $8 / 12$ tuvieron compromiso renal con raquitismo hipofosfémico, 2 presentaron 1 episodio de neuropatía, observándose en los 2 pacientes diagnosticados en edad escolar deformidades como xifoescoliosis y tórax en quilla. Un caso diagnosticado en edad escolar presentó fotofobia, siendo hospitalizada 2 veces por episodios catalogados como síndrome de Guillain Barre requiriendo en el primer episodio ventilación mecánica. Otra niña tuvo neuropatía en relación a la suspensión brusca del tratamiento con NTBC. La paciente diagnosticada a los 10 años debutó 6 años después con un hepatocarcinoma siendo trasplantada exitosamente en julio de 2009.

\section{Evolución después del tratamiento con NTBC}

Todos los pacientes presentaron buen control metabólico después del inicio de tratamiento, con descenso de ácido 5 aminolevulínico y succinilacetona a la semana de tratamiento hasta hacerse casi indetectable (Figuras 2 y 3 ). Se observó descenso de $\alpha$-feto proteínas en todos los casos a los 6 meses de tratamiento, pero en relación a la aparición de hepatocarcinoma en la paciente 2 se produjo gran aumento de $\alpha$-feto proteínas (Figura 4 ). La protrombina ascendió a niveles normales, observando esta normalización dentro de los 6 primeros meses de tratamiento y manteniéndose en el tiempo. La hipoprotrombinemia había sido tratada con vitamina $\mathrm{K}$ en todos antes del inicio de NTBC, no respondieron a esta terapia. Tres casos presen- 


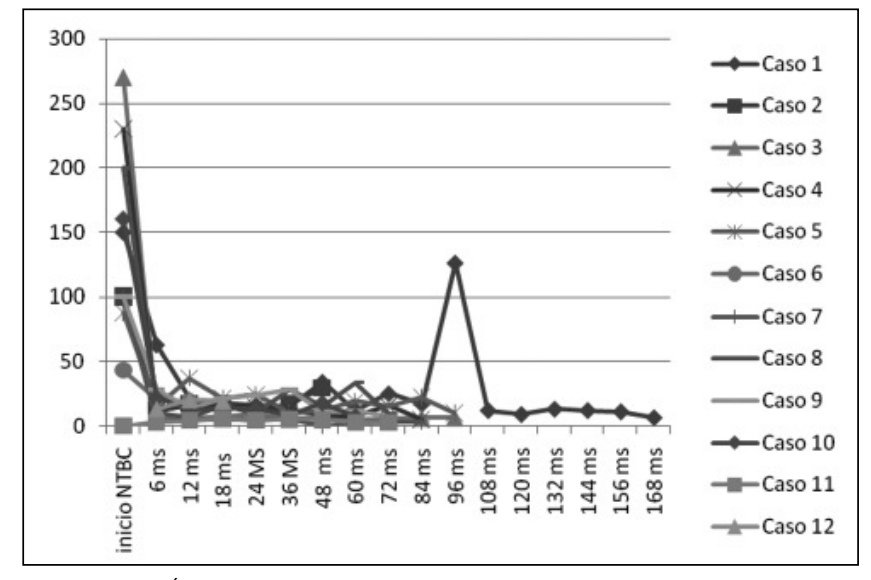

Figura 2. Ácido 5-aminolevulínico en niños con tirosinemia tipo I, tratados con NTBC $(n=12)$.

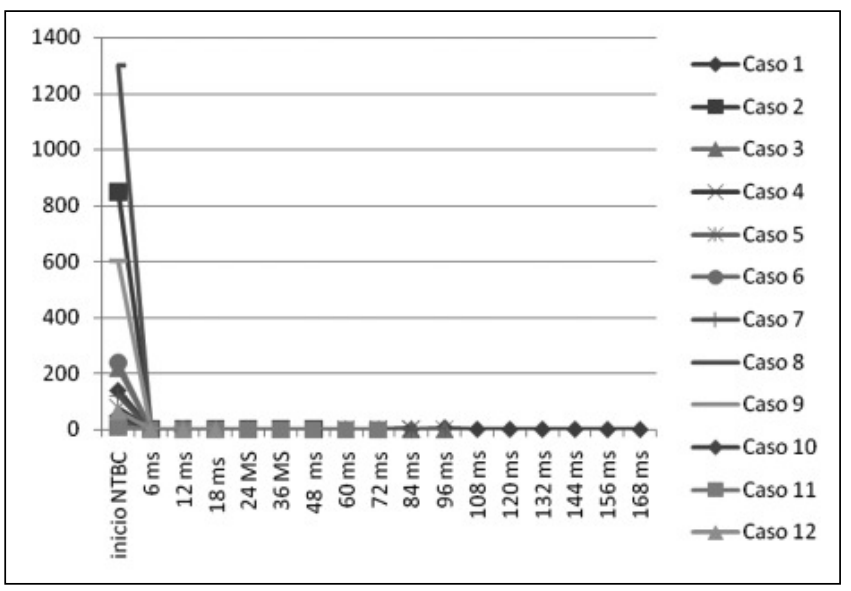

Figura 3. Succinilacetona en orina de niños con tirosinemia tipo 1 tratados con NTBC $(n=12)$.

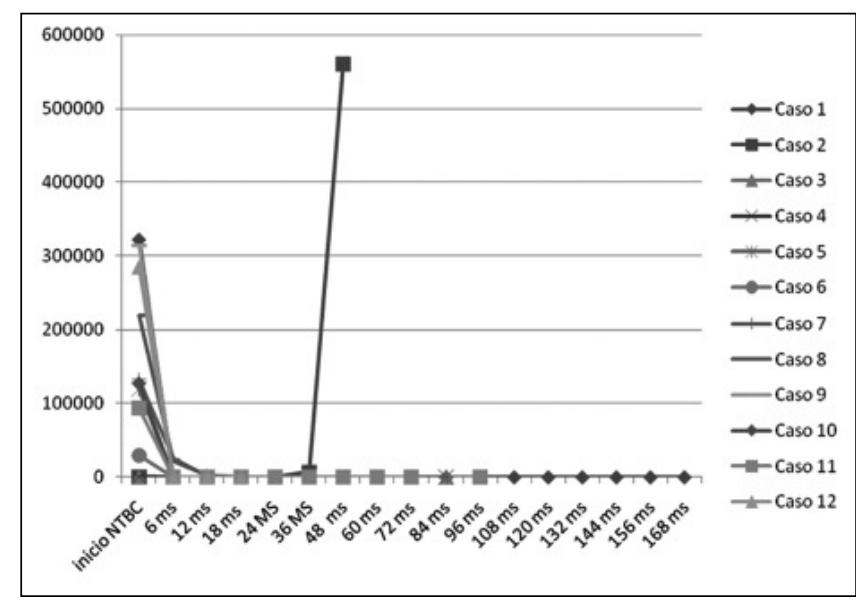

Figura 4. $\alpha$ Feto proteína en niños con tirosinemia tipo l, tratados con $\operatorname{NTBC}(n=12)$. taron descompensaciones metabólicas intercurrentes, objetivadas con aumento de ácido 5 aminolevulínico (Figura 2) y tirosina, secundarias a suspensión aguda de tratamiento farmacológico y nutricional. Los síntomas acompañantes fueron neuropatía periférica y fotofobia, llegando una paciente a requerir ventilación mecánica, seguido de deterioro del nivel intelectual. Los niveles de NTBC se han mantenido en rangos aceptables, entre 20 y $50 \mathrm{mMol} / \mathrm{L}$, salvo en el caso 1 que suspendió el tratamiento (Figura 5). En el paciente 3 que debutó con insuficiencia hepática aguda, se observó a los 3 meses de tratamiento cirrosis hepática en ultrasonografía abdominal, imagen que regresó al año de tratamiento.

\section{Estado clínico actual}

De los 12 casos seguidos, 2 fallecieron; una escolar que inició tratamiento a los 4 años y después de 4 años tuvo hepatocarcinoma con metástasis y otra falleció después de trasplante hepático. Cabe destacar que la primera paciente inició tratamiento a los 4 años de vida, habiendo estudios que sugieren mejor pronóstico cuando el tratamiento se inicia antes de los 2 años de vida ${ }^{12}$. La otra niña falleció después de trasplante hepático por hemorragia pulmonar como complicación de éste. Una paciente fue trasplantada a los 17 años y se curó de tirosinemia tipo 1. La condición clínica de los restantes 9 casos a junio 2010 se muestra en tabla 1.

El estado nutricional muestra: 8/12 son eutróficos, $1 / 12$ es eutrófico pero con talla baja, por raquitismo al momento del diagnóstico y el inicio tardío del tratamiento. En el desarrollo psicomotor: en $5 / 12$ fue normal y 4 escolares presentan retraso mental leve. En la función hepática y control metabólico, los 9 pacientes presentan buen control, demostrado con niveles normales de tirosina, fenilalanina y metionina, succinilacetona indetectable en orina, y buena función hepática y renal.

La joven que presentó neuropatía durante su evolución, se encuentra en 


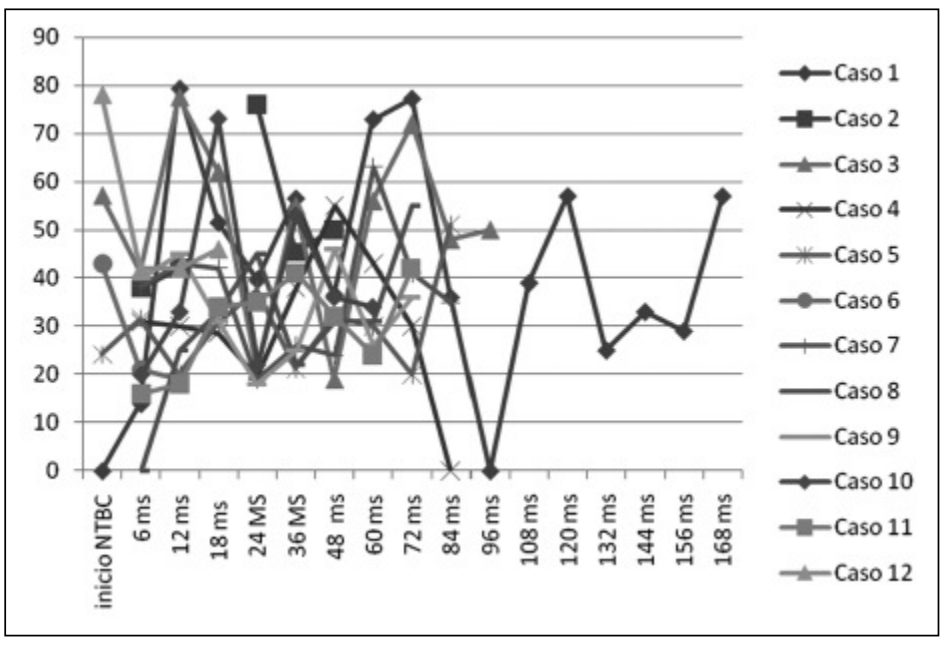

Figura 5. Niveles de NTBC en plasma en tirosinemia tipo I $(n=12)$. rehabilitación. No fueron reportadas reacciones adversas al NTBC durante el seguimiento.

\section{Discusión}

En los 12 pacientes seguidos se logró un excelente y rápido control metabólico, haciéndose indetectable succinilacetona y succinilacetato a la semana de tratamiento con NTBC. Estos resultados son concordantes con lo descrito por Holme y Lindstedt en $1998^{12}$ y otros autores ${ }^{13,1}$. Se ha mantenido el control metabólico en el tiempo y las descompensaciones se debieron a demoras en el ingreso de la droga al país. La sobrevida observada y la calidad de vida son excelentes, considerando que en el grupo de presentación muy temprana $(<2$ meses) se describe una supervivencia a los 2 años del 29\%. En este grupo se encuentran 4 casos de 14 años, 8 años 6 meses, 5 años 3 meses y 4 años 1 mes, los cuatro en buenas condiciones ${ }^{12}$. En el grupo de presentación temprana (2-6 meses) se describe supervivencia del 74\%. Existen 3 niños en este grupo dos de 6 años 6 meses y uno de 1 año 6 meses, en muy buenas condiciones. Hay 5 pacientes en el grupo de presentación crónica $(>6$ meses). En este grupo se encuentra la niña que murió por el hepatocarcinoma y la que falleció por el trasplante, asimismo la joven que fue exitosamente trasplantada a los 17 años por presentar un hepatocarcinoma. Además hay una niña de 11 años 7 meses y un niño de 6 años, ambos en muy buenas condiciones. De los 9 pacientes vivos, 5 presentan coeficiente intelectual normal. Los que han tenido una peor evolución desde el punto de vista cognitivo son los de diagnóstico más tardío y con descompensaciones posteriores. Sólo dos casos presentaron fracaso de tratamiento después de 4 años de iniciarlo, manifestado por aparición de hepatocarcinoma. Cabe destacar que una niña fue diagnosticada a los 4 años 9 meses, habiendo presentado síntomas desde el año de vida. La otra paciente comenzó el tratamiento a los 10 años, teniendo sintomatología desde los 8 meses de vida. En la publicación de Holme ${ }^{12}$ de los 101 pacientes que recibieron NTBC antes de los 2 años, ninguno desarrolló hepatocarcinoma a los 2 años de tratamiento, lo que concuerda con lo descrito por otros autores ${ }^{14,15} \mathrm{y}$ lo observado en este estudio.

El seguimiento de los pacientes chilenos demuestra que el uso de NTBC es una alternativa válida y exitosa al trasplante hepático en los casos con tirosinemia tipo I permitiendo dejar éste para aquellos afectados en que haya un fracaso a la respuesta terapéutica, con aparición de hepatocarcinoma como ocurrió en 1 caso el año 2009.

Finalmente, destacamos la importancia de la sospecha precoz en tirosinemia tipo I, dadas las diferencias dramáticas en tiempo y calidad de sobrevida en pacientes tratados y diagnosticados precozmente. Adherimos fuertemente a la propuesta de la Academia Americana de Pediatría sobre pesquisa neonatal, considerando su costo versus sobrevida en esta enfermedad actualmente tratable. Sería una medida aconsejable para usar en Chile, como ya se hace en otros países ${ }^{16-19}$. En América Latina sólo hay 2 países, Uruguay y Cos- 
ta Rica, que realizan pesquisa neonatal para esta enfermedad, pero aún no han encontrado $\operatorname{casos}^{20}$.

\section{Referencias}

1. Colombo M, Cornejo V, Raimann E. Errores innatos en el metabolismo del niño, Editorial Universitaria 2010; capítulo 3, p 71-138.

2. Protocolos para el diagnóstico y tratamiento de tirosinemia tipo I o hepatorrenal. An Esp Pediatr 2000; 53 (S2): 10-5.

3. Colombo M, Cornejo V, Raimann E. Errores innatos en el metabolismo del niño, Editorial Universitaria 2010; capítulo 22, p 692-6.

4. Jorquera R, Tanguay R. Fumarylacetoacetate, the metabolite accumulating in hereditary tyrosinemia, activates the ERK pathway and induces mitotic abnormalities and genomic instability. Hum Mol Genetics 2001; 10: 174152.

5. Van Spronsen FJ, Tomase Y, Smit GPA, Leonard JV, Clayton PT, Fidler V. Hereditary tyrosinemia type I: a new clinical classification with different prognosis on dietary treatment. Hepatology 1994; 20: 1187-91.

6. Dehner LP, Snover DC,Sharp HL, Ascher N, Nakhleh R, Day DL. Hereditary tyrosinemia type I (chronic form): pathologic findings in the liver. Hum Pathol 1989; 20: 149-58.

7. Van Dyk E, Steenkamp A, Koekemoer G, Pretorius PJ. Hereditary tyrosinemia type 1 metabolites impair DNA excision repair pathways. Biochem Biophys Res Commun 2010; 401(1): 32-6.

8. Lindstedt S, Holme E, Lock EA, Hjalmarson O, Strandvivik $B$. Treatment of hereditary tyrosinemia type 1 by inhibition of 4-hydroxyphenylpyruvate dioxygenase. Lancet 1992; 340: 813-817.

9. Joshi S N, Venugopalan P. Experience with NTBC therapy in hereditary tyrosinemia type I: an alternative to liver transplantation. Ann Trop Pediatr 2004; 24: 259-65.
10. Holme E, Lindstedt S. Diagnosis and management of tyrosinemia type 1. Curr Opin Pediatr 1995; 7: 726-32.

11. Holme E, Lindstedt S, Lock EA. Treatment of tyrosinemia type 1 with an enzyme inhibitor. Int Pediatr 1995; 10: 41-3.

12. Holme E, Lindstedt S. Tyrosinemia type 1 and NTBC (2(2-nitro-4- trifluoromethylbenzoyl)-1, 3-cyclohexanedione). J Inher Metab Dis. 1998; 21: 507-17.

13. Del Hoyo Gil L, Serrano O, Ferrari J.M, Medina e, Herreros A. NTBC en el tratamiento de la Tirosinemia Tipo I: descripción de un caso. Farm Hosp 1997; 21: 324-6.

14. Crone J, Moslinger D, Bodamer OA, Schima OA, Huber WD, Holme E, et al. Reversibility of cirrhotic regenerative liver nodules upon NTBC treatment in a child with tyrosinemia type I. Acta Paediatr 2003; 92: 625-8.

15. El-Karaksy H, Rashed M, El-Sayed R, El-Raziky M, ElKoofy N, El-Hawary M, et al. Clinical practice. NTBC therapy for tyrosinemia type 1: how much is enough? Eur J Pediatr 2010; 169 (6): 689-93.

16. Kaye CI, and the Committee on Genetics. Newborn Screening Fact Sheets. Pediatrics 2006; 118: 934-63.

17. Turgeon C, Magera MJ, Allard P, Tortorelli S, Gavrilov $\mathrm{D}$, Oglesbee D, et al. Combined newborn screening for succinylacetone, amino acids, and acylcarnitines in dried blood spots. Clin Chem 2008; 54 (4): 657-64.

18. Schlump JU, Mayatepek E, Spiekerkoetter U. Significant increase of succinylacetone within the first $12 \mathrm{~h}$ of life in hereditary tyrosinemia type 1. European J Pediatr 2010; 169 (5): 569-72.

19. La Marca G, Malvagia S, Funghini S, Pasquini E, Moneti G, Guerrini R, et al. Letter to the Editor: The successful inclusion of succinylacetone as a marker of tyrosinemia type 1 in Tuscany newborn screening program. Rapid Communications in Mass Spectrometry 2009; 23: 38913 .

20. Borrajo GJ. Newborn screening in Latin America at the beginning of the $21^{\text {st }}$ century. J Inherit Metab Dis 2007; 30 (4): 466-81. 